\title{
Effects of Diverse Connecting Mechanisms on Knowledge Diffusion
}

\author{
Xiaorong Chen Jin Ding Haoyuan Zhou \\ Antai College of Economics \& Management, Shanghai Jiao Tong University Shanghai 200052, P.R. China
}

\begin{abstract}
In this paper, we first propose a computational model. Then on the basis of two virtual knowledge networks constructed, we investigate how diverse connecting mechanisms between network A and B differentially influence the co-evolution of the knowledge levels of both networks. The simulation results indicate that agents with high brokerage opportunities or high centrality enhance their knowledge levels more rapidly and knowledge diffusion through different knowledge networks seems to be affected significantly by the characteristics of the agents who build the knowledge connections across these networks.
\end{abstract}

Keywords: Complex knowledge network, Bottom-Up simulation modeling, Knowledge diffusion, Centrality, Brokerage

\section{Introduction}

The last few years have witnessed a tremendous activity devoted to the characterization and understanding of networked systems. There is a growing consensus among socio-economic scientists that many socio-economic phenomena display some inherent network features. Studies on these phenomena should not exclude the consideration of the role of the network structure.

The study of network structural effects on knowledge diffusion has attracted considerable interests [1]-[3]. Reference [1] and [2] examined the relationship between network architectures and diffusion performance and pointed out that when a network structure is a small world, the steady-state level of average knowledge is maximal. In [3], simulation results showed that interregional network structures interact with the observability of an innovation's benefits to determine diffusion. One common point of these research is that they utilized computational simulation experiments.

One of the limitations of the existing research utilizing computational simulation experiments is that the network models, on which simulations were conducted, were very simple, such as regular network model, small world model, and etc. However, several empirical studies [4]-[7] have reported some important properties of social networks, such as small-world effects, an approximately scale-free degree distribution, a positive correlation in degree between neighboring nodes and a negative relationship between node degree and local clustering.

Complex knowledge networks are typical social networks, which include many agents like organizations, persons and etc. The typical characteristic of social networks is that they consist of many active agents with bounded rationalities. Different from the existing literature, we construct virtual knowledge networks, utilizing a network formation model proposed by [8]. This was the first model of network formation that accounts for five features that characterize large socially generated networks: (i) a small diameter, (ii) high clustering, (iii) an approximately scale free degree distribution, (iv) a positive correlation in degree between neighboring nodes, and (v) a negative relationship between node degree and local clustering.

The second limitation of the existing research utilizing computational simulation experiments is that the local network structural effects on knowledge diffusion have received minimal attention. By contrast, many empirical studies focused on this issue [9]-[12]. Reference [9] pointed out that strong ties facilitate both explicit knowledge and tacit knowledge. Reference [10], [11] found a correlation between key intellectual properties and the centrality of actors. Reference [12] showed that people, acting as brokers with connections across structural holes, have competitive advantages in seeing and developing good ideas. In this paper, we are aiming to investigate the local network structural effects on knowledge diffusion, and explore several connecting mechanisms in order to enhance the knowledge level of a knowledge network.

The outline of this paper is as follows. In Sect.2, we first propose a computational model for the knowledge network. Then on the basis of this model, simulation experiments are conducted and the results 
are given in Sect.3. In Sect.4, we give some brief conclusions and reveal managerial implications.

\section{The model}

In the following, we firstly construct a virtual network consisting of $N$ nodes using Jackson's network formation model. Jackson's model is based on an explicit search process. Let $N_{t}$ denote the set of all nodes present at time $t$. At each time $t$, a new node is added to the population. Upon birth, the new node identifies $M_{r}$ nodes uniformly at random from $N_{t-1}$. We call these nodes parent nodes. Let $P_{r}$ denote the probability that a new node finds a randomly identified node attractive to link to. In addition, the node searches the parent's immediate neighborhood and finds other nodes. Let $P_{s}$ denote the probability that the new node obtains a positive utility from linking to a given node found through search.

Next, on the basis of a constructed virtual network, we model behavior of agents in the network. Then following the basic idea of building a system from Bottom to UP, a structural realistic model of a knowledge network is proposed. It is worth to note here that we adopt a strategy called pattern-oriented modeling [13], which attempts to make bottom-up simulation modeling more rigorous and comprehensive. Patterns are defining characteristics of a system, and often therefore indicators of essential underlying processes and structures. We found some patterns, which seem to characterize a knowledge network and its dynamics, and then implemented these patterns in our simulation model. This helps us optimize model complexity and reduce uncertainty.

In a network, each agent is represented with a node. Agents could exploit external knowledge sources within or across regions through formal knowledge connections (such as technological alliance) or informal ones (such as technological communication). If there are connections between two agents, two nodes representing these two agents will be linked by an edge. Two agents interact if and only if there is a direct connection between them.

Agent $i$ is characterized by a certain amount of knowledge endowment, denoted as $K_{i}$, which indicates the knowledge stock level of this agent. We define the degree of agent $i$ as the number of direct connections of agent $i$ has, denoted as $D_{i}$. And the degree of agent $i$ is regarded as the indicator of its centrality, however, there are three measures of centrality, namely degree centrality, betweeness centrality, closeness centrality. Degree centrality is a common measure of the centralization of power in organized studies. In general, one can argue that agents with more relationships with the others have higher levels of centrality. It is assumed that the degree and the knowledge stock level of an agent have no correlation.

If there exists an edge between node $i$ and node $j$, we denote the weight of this edge as $W_{i j}$, which indicates the intensity of this relationship. Here, we assume the weight of an edge is following a uniform distribution $U(0,1)$. If the weight of an edge is set to be 1 , this indicates the strongest connection between the corresponding nodes. On the other hand, that the weight of an edge is assigned a value of 0 indicates no connection between the corresponding nodes.

In this paper, we use network constraint to measure brokerage. Let $p_{i j}$ denote the proportion of agent $i$ 's network time and energy invested in contact $j \cdot N C_{i j}$ measures to what extent agent $i$ 's network is directly or indirectly investing in the agent's relationship with contact $j$. And $N C_{i}$ indicates the network constraint of agent $i$. In general, one can argue that agents with low levels of network constraint have higher levels of brokerage opportunities [14].

$$
\begin{gathered}
p_{i j}=\frac{w_{i j}}{\sum_{q} w_{i q}} \\
N C_{i j}=\left(p_{i j}+\sum_{q} p_{i q} p_{q j}\right)^{2} \quad(q \neq j) \\
N C_{i}=\sum_{q} N C_{i j}
\end{gathered}
$$

An agent is characterized by a certain knowledge absorptive capacity, which determines to what extent that the knowledge it receives from an external source can be assimilated. Reference [15] suggested that a firm's absorptive capacity is a function of the firm's level of prior related knowledge. Therefore, we assume in this paper that the absorptive capacity of agent $i$, denoted as $C_{i}$, increases with $K_{i}$ as follows

$$
C_{i}=\frac{1}{1+e^{-\alpha_{1} K_{i}}}
$$

where parameter $\alpha_{1}$ controls the speed to access the upper limit 1.

Next, we assume the knowledge stock of each agent consists of two parts. One part increases endogenously with the continual $\mathrm{R} \& \mathrm{D}$ expenditure. The second part is the part flowing into the agent through external connections, so-called the exogenous part. We give the expression of the knowledge stock level of agent $i$ at time $t+1$, denoted as $K_{i}(t+1)$, as follows 


$$
K_{i}(t+1)=K_{i}(t)+\Delta K_{i-i n}(t)+\Delta K_{i-o u t}(t)
$$

where $\Delta K_{i-i n}(t)$ indicates the endogenous part and $\Delta K_{\text {i-out }}(t)$ indicates the exogenous part respectively.

We assume that $\Delta K_{i-i n}(t)$ increases according to following model. Let parameter $\beta$ control the upper limit, while letting parameter $\alpha_{2}$ control the speed to access that upper limit.

$$
\Delta K_{i-i n}(t)=\frac{\beta K_{i}(t)}{1+e^{-\alpha_{2} K_{i}(t)}}
$$

Let $\Delta K_{\text {i-out }}(t)$ be calculated with the following algorithm. Here, $U_{i}$ indicates the set consisting of all the nearest neighbors of agent $i$. At every $\Delta t$ time period, agent $i$ searches all its nearest neighbors one by one.

For example, considering the adjacent agent $j\left(j \in U_{i}\right)$, only if the knowledge stock level of agent $j$ is higher than that of agent $i$, agent $i$ is given an opportunity to get its knowledge stock level enhanced. The increment of its knowledge stock level equals to the knowledge stock level difference between these two agents times the mediating factor $C_{i}(t)^{*} W_{i j}$. Obviously, the weight of an edge determines to what extent this agent can receive the counterpart's knowledge through a connection. Meanwhile, the absorbing capacity of this agent determines to what extent this agent can absorb the received knowledge. Then based on the new knowledge stock level of agent $i$, the next agent in $U_{i}$ would be processed. This learning process continues until all learning possibilities are exhausted.

For this economy, we measure the aggregate performance of a network by observing the total average knowledge level over all agents, denoted as $T A K(t)$. Let $T A K(t)$ be represented as

$$
\operatorname{TAK}(t)=\frac{\sum_{i=1}^{N} K_{i}(t)}{N}
$$

where $N$ indicates the number of agents in the network.

\section{Numerical analysis}

\subsection{Independently developing network}

Initially, we design a virtual knowledge network, consisting 500 nodes. Here, TAK indicates the total average knowledge level of this network. Within a single simulation experiment, we let the focal network develop independently, and evolve over 1000 time periods. The initial value of $T A K$ is set as TAK $\sim U(a, b)$. Per time period, $\operatorname{TAK}(t)$ is recorded respectively.

In simulation experiments, all parameters are assigned values as follows:

$$
\begin{gathered}
\alpha_{1}=0.001 \quad \beta=0.005 \quad \alpha_{2}=0.001 \\
a=1 \quad b=1000
\end{gathered}
$$

Firstly, we target the following three groups of agents.

(group 1)agents with initially high knowledge levels. (group 2)agents with high brokerage opportunities. (group 3)agents with high centrality.

Then we investigate the evolution of knowledge levels of these three groups of agents. Fig.1 shows the simulation results. We could see that, all three groups of agents show above average speed of knowledge enhancement. Moreover, the knowledge levels of group 2 and group 3 catch up with that of group 1 at some time period. The agents in group 2 and group 3 learn very quickly, taking great advantage of their important structural positions in the focal network, however, they might not have the highest knowledge levels at the beginning.

Here, since we referred Burt's idea of measuring brokerage opportunities, we found that the centrality of agents is highly positively correlated with their brokerage opportunities. Therefore, we could not differentiate the behavior of group 2 and group 3, however, in some cases of loosing cohesive networks, group 2 and group 3 behave differently.

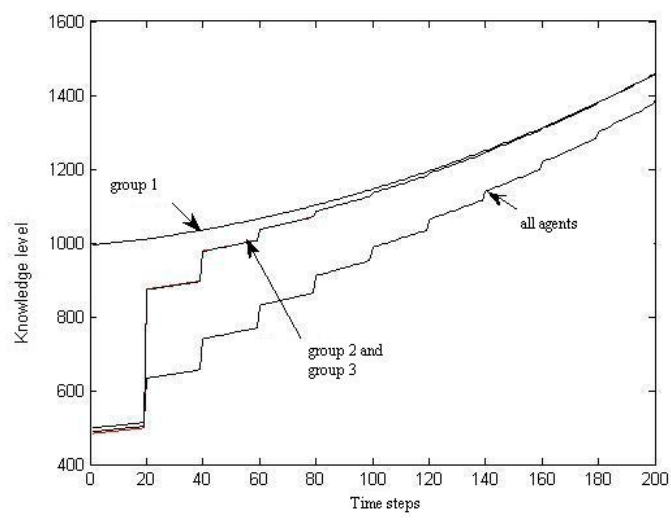

Fig. 1: Evolution of knowledge levels of three groups.

\subsection{Co-evolving networks with bridging ties}

Initially, we design two virtual knowledge networks $A$ and $B$, both consisting 500 nodes and having a distinct difference in their total average knowledge levels. Here, $T A K_{A}$ indicates the total average 
knowledge level of network $A$ and $T A K_{B}$ indicates the total average knowledge level of network $B$ respectively. The initial values of $T A K_{A}$ and $T A K_{B}$ are set as

$$
\text { TAK }{ }_{\text {A }} \sim U\left(b_{1}, b_{2}\right) \text { TAK }{ }_{B} \sim U\left(b_{3}, b_{4}\right)
$$

where $b_{3}<b_{1}<b_{4}<b_{2}$ is assumed to ensure a distinct difference in total average knowledge levels between two networks.

Within a single simulation experiment, we let two virtual knowledge networks $A$ and $B$ evolve over 1000 time periods. Per time period, $T A K_{\mathrm{A}}(t)$ and $T A K_{\mathrm{B}}(t)$ are recorded respectively.

In simulation experiments, all parameters are assigned values as follows:

$$
\begin{gathered}
\alpha_{1}=0.001 \quad \beta=0.005 \quad \alpha_{2}=0.001 \\
b_{1}=400 \quad b_{2}=1000 \quad b_{3}=0 \quad b_{4}=600
\end{gathered}
$$

Our aim is to investigate whether several diverse connecting mechanisms between different networks could help enhance the performance of the less developed knowledge network (network B).

\subsubsection{Invariant bridging ties}

At the beginning of a simulation experiment, we firstly target some agents with specified characteristics from both network $A$ and network $B$, then let them build connections among them. These bridging ties will remain invariant through the whole simulation time window.

In the following, five cases are investigated.

(Case 0) There are no connections between network A and network B.

(Case 1) Some agents in network A with higher knowledge levels build connections with some agents in network B with higher knowledge levels.

(Case 2) Some agents in network A with higher centrality build connections with some agents in network B with higher centrality.

(Case 3) Some agents in network A with higher brokerage opportunities build connections with some agents in network B with higher brokerage opportunities.

(Case 4) Some randomly selected agents in network A build connections with some randomly selected agents in network $\mathrm{B}$.

Fig. 2 shows the simulation results of network B. As we could see, obviously the knowledge level of network $B$ in the case of no connections between network A and network B (case 0) is the lowest at each time period, compared with the other cases with the existence of connections. The knowledge level of network B in case 1 behaves similarly to that of case 4 , which is lower than that of case 2 and case 3 .
Here, we found that the centrality of agents is highly positively correlated with their brokerage opportunities, which might answer the question why the knowledge level of network B in case 2 behaves similarly to that in case 3 . From the simulation results, we could draw the conclusion that the connecting mechanism, namely purposely connecting some targeted agents with high centrality or brokerage opportunities from both network $\mathrm{A}$ and $\mathrm{B}$ will contribute to the rapidest enhancement of the knowledge level of network B.

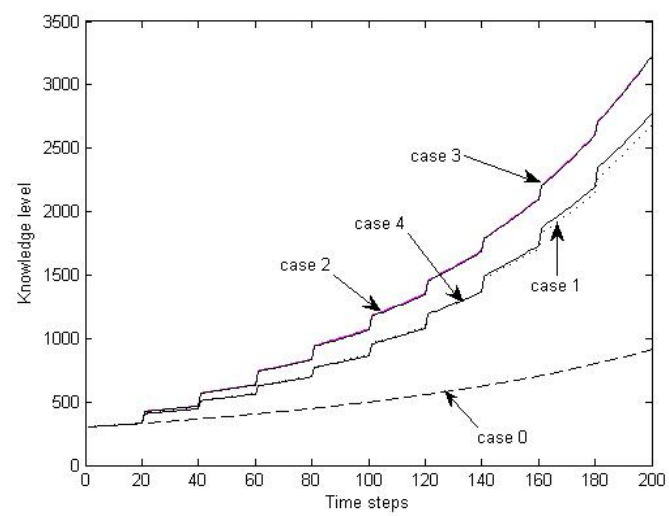

Fig. 2: Evolution of knowledge levels of five cases.

In addition, Fig.3 shows the comparison of evolution of knowledge levels of network $A$ and network B in case 0 and case 3 . We could see that, while evolving with time periods, the gap of the knowledge levels of two networks in case 3 is maintained at an acceptable level, while in case 0 the gap has a tendency to be enlarged gradually.

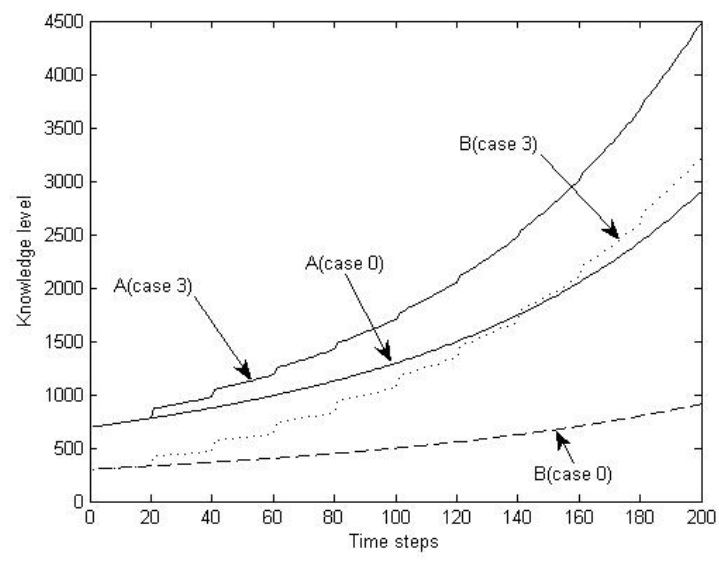

Fig.3: Comparisonbb of knowledge levels (network A and B).

\subsubsection{Dynamically rebuilding bridging ties}

Next, we investigate several other connecting mechanisms. At each time period similarly we target some agents with specified characteristics from both 
network $\mathrm{A}$ and network $\mathrm{B}$, then let them build connections among them. But in each connecting mechanism, these bridging ties will be rebuilt dynamically.

In the following, three cases are investigated.

(Case 5-1) Targeting the agents with both high knowledge levels and high brokerage opportunities from some selected agents with high knowledge levels in network A and network B, then building connection among them.

(Case 5-2) Targeting the agents with both high brokerage opportunities and high knowledge levels from some selected agents with high brokerage opportunities in network A and network B, then building connection among them.

(Case 5-3) Selecting some agents with high knowledge stock levels in network A and network $\mathrm{B}$, then building connections among them.

Fig. 4 shows the simulation results of network B. As we could see, the knowledge level of network B in case 5-3 ranks the lowest, compared with the other cases. Case 5-1,5-2 and case 3 (or case 2, defined in section 3.2.1) behave similarly. Moreover, the knowledge level of network B in case 5-2 ranks the highest, however, the difference from that of case 3 is not significant.

We could reach a conclusion that, in order to enhance the knowledge level of a less developed knowledge network, the optimum connection mechanism is what is proposed in case 5-2. However, dynamically rebuilding bridging ties seems very costly. Therefore, we recommend the connecting mechanism proposed in case 3 or case 2, namely selecting some nodes in network A and network B with high brokerage opportunities or high centrality, building connections among these nodes, and keeping the bridging ties invariant with time $t$, which has the satisfied effectiveness, but not so costly.

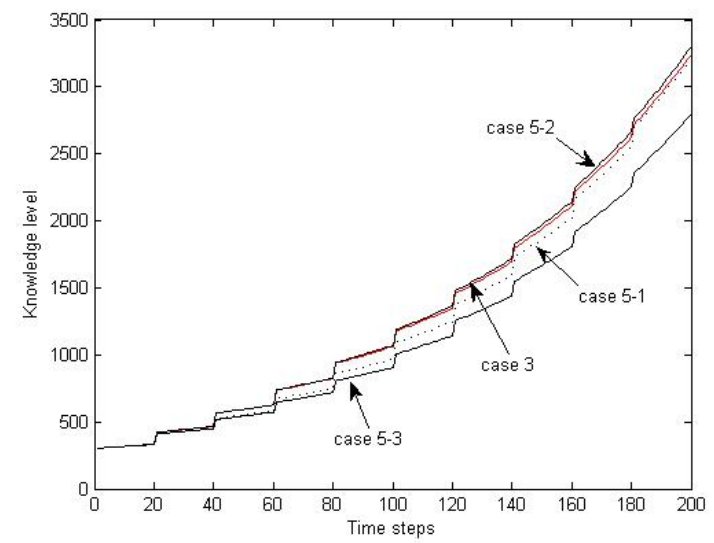

Fig. 4: Evolution of knowledge levels of four cases.

\section{Conclusions}

In this paper, we first propose a computational model. Then on the basis of two virtual knowledge networks constructed, we investigate how diverse connecting mechanisms influence the evolution of the knowledge levels of these networks. Based on the simulation results, we could draw the following two conclusions.

Firstly, agents with high brokerage opportunities or high centrality enhance their knowledge levels more rapidly, which draws a good line with the empirical research [10-12].

Secondly, knowledge diffusion through different knowledge networks seems to be affected significantly by the characteristics of the agents who build the knowledge connections among networks, namely high knowledge levels, high centrality and high brokerage opportunities. We made a comparative analysis of several different connecting mechanisms, among which the mechanisms of building connections among the agents with high brokerage opportunities or high centrality behave the best.

This research could provide guidance for policymakers who would rather speed knowledge diffusion through a knowledge network. Through purposely building knowledge connections among the agents with high brokerage opportunities or high centrality in different networks, the knowledge diffusion through different knowledge networks is the most effective. Policy-makers should target the agents with high brokerage opportunities or high centrality in both the less developed knowledge network and the high developed knowledge network first, and then encourage the connections among these agents, rather than let the knowledge networks self-organize their structures.

Finally, there are three limitations of this research. Firstly, we studied the structural effects of diverse connecting mechanisms on knowledge diffusion, on the basis of a comparatively static network structure. However, system dynamics and the underlying topology are mutually correlated. Network structure is also evolving, being affected by the various dynamical processes. Secondly, our assumption that knowledge networks have some typical characteristics as the other types of social networks have, which need to be demonstrated empirically. Thirdly, the modeling of the behavior of agents should take their adaptive learning abilities into consideration.

\section{Acknowledgement}

This work is supported by the National Natural Science Foundation of China (Grant No. 70401019). 


\section{References}

[1] R. Cowan, N. Jonard, The Dynamics of Collective Invention. Journal of Economic Behavior \& Organization, 52:513-532, 2003.

[2] R. Cowan, N. Jonard, Network Structure and the Diffusion of Knowledge. Journal of Economic Dynamics \&Control, 28:1557-1575, 2004.

[3] Deborah E. Gibbons, Network Structure and Innovation Ambiguity Effects on Diffusion in Dynamic Organizational Fields. Academy of Management Journal, 47(6): 938-951, 2004.

[4] M. E. J. Newman, Assortative Mixing in Networks. Physics Review Letter, 89(20): 208701, 2002.

[5] M. E. J. Newman, The Structure and Function of Complex Networks. SIAM Review, 45: 167-256, 2003.

[6] M. E. J. Newman, J. Park, Why Social Networks Are Different from Other Types of Networks. Physical Review E, 68:036112, 2003.

[7] B.Verspagen, G. Duysters, The Small Worlds of Strategic Technology Alliances. Technovation, 24: 563-571, 2004.

[8] M.O. Jackson, B.W. Rogers, Search in the Formation of Large Networks: How Random Are Socially Generated Networks. American Economic Review, to appear.

[9] R. Reagans, B. McEvily, Network Structure and Knowledge Transfer: the Effects of Cohesion and Range. Administrative Science Quarterly, 48:240267, 2003.

[10]B. Gay, B. Dousset, Innovation and Network Structural Dynamics: Study of the Alliance Network of a Major Sector of the Biotechnology Industry. Research policy. 34:457-1475, 2005.

[11]W.W. Powell, K.W. Koput, L. Smith-Doerr, Interorganizational Collaboration and the Locus of Innovation: Networks of Learning in Biotechnology. Administrative Science Quarterly, 41(1): 116-145, 1996.

[12] Ronald S. Burt, Structural Holes, Good Ideas. The American Journal of Sociology, 110( 2): 349399,2004.

[13] V. Grimm, E. Revilla, U. Berger, et. al., PatternOriented Modeling of Agent-Based Complex Systems: Lessons from ecology. Science, 310:987-991, 2005.

[14] Ronald S. Burt, Structural Holes, Cambridge, Harvard University Press, 1992.

[15]W.M. Cohen, D.A. Levinthal, Absorptive Capacity: a New Perspective on Learning and Innovation. Administrative Science Quarterly, 35(1): 128-152,1990. 